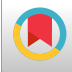

\title{
Survival Rate of Patients with Bladder Cancer in Yazd, Central Province of Iran
}

\author{
Hassan-Ali Vahedian-Ardakani, ${ }^{1}$ Mansour Moghimi, ${ }^{2}$ Mohammad Shayestehpour, ${ }^{3,4}$ Masoud Doosti, ${ }^{5}$ \\ and Fatemeh Alimohammadi ${ }^{6,}$ \\ ${ }^{1}$ Department of Internal Medicine, School of Medicine, Shahid Sadoughi University of Medical Sciences, Yazd, Iran \\ ${ }^{2}$ Department of Pathology, School of Medicine, Shahid Sadoughi University of Medical Sciences, Yazd, Iran \\ ${ }^{3}$ Department of Microbiology and Immunology, Faculty of Medicine, Kashan University of Medical Sciences, Kashan, Iran \\ ${ }^{4}$ Autoimmune Research Center, Kashan University of Medical Sciences, Kashan, Iran \\ ${ }^{5}$ Infectious and Tropical Diseases Research Center, Shahid Sadoughi University of Medical Sciences, Yazd, Iran \\ ${ }^{6}$ Aliebn- Abitaleb School of Medicine, Islamic Azad University, Yazd Branch, Yazd, Iran \\ "Corresponding author: Fatemeh Alimohammadi, Aliebn- Abitaleb School of Medicine, Islamic Azad University, Yazd Branch, Shohada-e-Gomnam Boulevard, Safaieeh, Yazd, \\ Iran. Tel/Fax:+98-351831872664, E-mail: h.vahedian@ssu.ac.ir
}

Received 2017 September 06; Revised 2017 December 05; Accepted 2018 March 05.

\begin{abstract}
Background: Bladder cancer is the ninth most commonly diagnosed malignancy worldwide. The trend of bladder cancer incidence and mortality is rising in Iran. This study was aimed to evaluate the survival rate of patients with bladder cancer in Yazd province, Iran.

Methods: In this retrospective cohort study, data were collected from 340 patients suffering from bladder cancer referred to Shahid Rahnemon and Shohada-Kargar Hospitals in Yazd province, Iran between April, 2001 and March, 2012. Variables included age, gender, stage of cancer, place of residence and type of treatment. The Kaplan-Meier and Cox regression analyses were used to evaluate the relationship between each variable and survival time. A P value less than 0.05 was considered significant.

Results: The mean age of total patients was $65.8 \pm 13.6$ years, and their mean survival time was $68.55 \pm 6.05$ months. Cumulative survival rates at the end of 1,3 , and 5 years in bladder cancer patients were $91 \%, 58 \%$, and $51.4 \%$, respectively. A statistically significant association was found between age $(\mathrm{P}=0.005)$, stage of disease $(\mathrm{P}=0.0003)$, type of treatment $(\mathrm{P}=0.0003)$ and survival time of patients. Data showed no significant correlation between age, gender, place of residence and patients' survival.

Conclusions: The survival of patients suffering from bladder cancer in this study was less than other reports. Patients'age and cancer stage were the effective factors in survival time. Continuous screening of older people for cancer diagnosis in early stages is seemed to improve survival in patients.
\end{abstract}

Keywords: Bladder, Cancer, Survival Rate, Iran

\section{Background}

Bladder cancer is a common cancer of urinary tract .The available statistics for bladder cancer show that this malignancy is the ninth leading cause of cancer in the world (1). The number of bladder cancer deaths has increased from 114,000 in 1990 to 170,000 in 2010 (2). In 2012, 430,000 new bladder cancer cases and 165,000 deaths occurred worldwide. About $60 \%$ of cases were diagnosed in more developed countries. The highest incidence of this cancer was in Northern America and Europe while the lowest incidence was in Asia and Latin America (3). Bladder cancer is the fourth most common cancer in Iranian men (4). Based on the available data, bladder cancer is the second, the third and the fifth common cancer in Markazi,
Hamedan and Fars provinces of Iran, respectively $(4,5)$.

Smoking is a serious risk factor for bladder cancer. Smoking causes about 50\% of all bladder cancers in women and men (6). Other risk factors include age, race, gender, chronic bladder infections, radiation exposure, bladder birth defects and genetic history (5). Bladder cancer mainly occurs in the population older than 65 years old. This cancer is about 3 - 4 times more prevalent in men than women $(4,7)$. Several studies were conducted to estimate the survival rate of bladder cancer in the world (4). According to the most recent data, the general 5-year relative survival rate for patients with bladder cancer (including all stages) is 77\%, and the 10-year relative survival rate is about $70 \%$. Survival rate of bladder cancer is dependent on metastasis to other organs, stage, grade and size of tumor 
(7). For patients with non-muscle invasive bladder cancer, the 5 -year survival rate is $96 \%$, while for patients with invasive tumor, the 5-year survival rate is $70 \%$. If the bladder tumor spreads to the surrounding tissues, lymph nodes or organs, the 5-year survival rate is 34\% (3).

Mortality and bladder cancer survival rates are known to vary considerably among countries in terms of public health care system, access to diagnosis and treatment facilities, and effective treatment protocols. Cancer survival statistics can be useful for determining prognosis and evaluating treatment options. Therefore, knowing about cancer survival rates in any country and its provinces/states is necessary (3). Although bladder cancer incidence has increased in the last 15 years in Iran, the survival rate of Iranian patients is not clear in all regions of the country (4). Published data on survival of patients with bladder cancer in Iran are really little. The present study aimed to determine, for the first time, the survival rate of patients with bladder cancer in Yazd province.

\section{Methods}

This retrospective cohort study was conducted on 340 patients with bladder cancer referred to Shahid Rahnemon and Shohada-Kargar Hospitals in Yazd, Iran between April, 2001 and March, 2012. The study was approved by the ethics committee of Shahid Sadoughi University of Medical Sciences-Yazd. Inclusion criteria were adult patients suffering from transitional cell bladder cancer and patients receiving chemotherapy or radiation treatment. Exclusion criteria were patients under 18 years and patients with non-transitional cell tumors. The underlying cause of death was obtained from the death certificates and patients' documents. The patients who died due to noncancer causes did not include the study. In order to gather data, in the first step, patient information documents were collected by referring to the cancer registry center of Shahid Rahnemon and Shohada-Kargar Hospitals. In the second step, the final status of the patients was checked by phone calls. In case of unsuccessful phone calls, patients were re-contacted by telephone at least three times a week. If the patients did not respond to the phone calls in a two-week period, they were not included in the study. The participants should be fully monitored to ensure their survival. Therefore, patients who were lost to follow up due to migration or changed their phone numbers were excluded from the study.

Data were collected by two questionnaires. The first one contained general information of patients, including demographic variables such as age, gender, stage of cancer, place of residence and type of treatment, which was completed using the patient's' medical records. The second questionnaire included the participants' follow-up information such as, the contact time and the patients' status.

Data were analyzed using SPSS software version 17 (Chicago, IL, USA). Qualitative variables were reported as proportions and continuous variables as mean \pm SD. Kaplan-Meier analysis was used to estimate survival in patients with bladder cancer. Log-rank test was performed to compare the survival experience of two (or more) groups. A Multivariate analysis was performed using the Cox proportional hazard regression model. A P value less than 0.05 was considered statistically significant.

\section{Results}

In this study, 340 patients with bladder cancer were followed up of whom 156 patients (45.9\%) were alive and 184 cases (54.1\%) had died due to cancer. Distribution of patients with bladder cancer according to different variables is summarized in Table 1 . Of all patients, 300 cases (88.2\%) were men, and 40 cases (11.8\%) were male. The mean age of total patients was $65.8 \pm 13.6$ years. Furthermore, mean age for men and women was $64 \pm 12.6$ and $68 \pm 14.4$ years, respectively. The mean and median survival time in patients were $68.55 \pm 6.05$ and 51 months, respectively. In this study, men had a higher survival time mean than women, but the difference was not significant $(\mathrm{P}=0.33$, Table 1). Five-year bladder cancer survival rate was $51.4 \%$ for all patients and was 51.5 and 50\% for men and women, respectively. The patients were divided into two age group, 30 - 69 and 70 - 89 year old. The highest number of patients was in the age group 30 - 69 year old (Table 1). Patients in age group 70 - 89 had significantly worse survival rate than that age group $30-69(\mathrm{P}=0.005$, Table 1$)$. In the present study, the cancer survival time was estimated for patients in different stages of bladder cancer. People suffering from bladder cancer stage 0 and 1 had the best survival while the worst survival was seen in patients with bladder cancer stage 4 (Table 1 ). Fifty nine cases were treated by transurethral resection of bladder tumor (TURBT). These patients had a longer survival period than those treated by partial cystectomy or radical cystectomy. Data obtained from comparing patients treated and untreated with radiotherapy showed that radiation therapy decreased survival time in patients. Unlike intravesical chemotherapy, systemic chemotherapy had a reducing effect on survival time of patients $(\mathrm{P}=0.0002)$. Results of multivariate survival analysis using Cox regression method are shown in Table 2. This data revealed that age, cancer stage and type of treatment play an important role in survival of bladder cancer patients. Patients in age range of 30 - 69-year-old 
had the risk of bladder cancer 29.3 time more compared to those between 70 and 89 . The following parameters significantly increased HR for mortality risk of bladder cancer: bladder cancer stage IV, partial cystectomy and radical cystectomy. Systemic chemotherapy increased probability of death in bladder cancer patients (HR: 0.28, Table 2).

\section{Discussion}

The present study was designed to evaluate, for the first time, the survival rate in patients with bladder cancer in Yazd, Iran. The data analyses showed that the overall survival rate at the end of one, three and five years were $91 \%$, $58 \%$, and $51.4 \%$, respectively. In the study by Manterola et al. conducted in Chile, the five-year survival rate for patients with bladder cancer was reported as 50\% (8). Sankaranarayanan et al. estimated that the five-year survival rate for bladder cancer in China, Republic of Korea, Turkey, Singapore was 63\% - 76\% (9). Another study reported that the five-year survival rate of bladder cancer was $61,66,63$, 59, and 52\% in Caucasian, Japanese, Chinese, Filipino, and Hawaiian patients, respectively (10). In the present study, the survival rate of patients with bladder cancer in Yazd city of Iran is lower than the mentioned areas. In the study by Shamsnia and colleagues in Shiraz city, the bladder cancer survival rates were $94.40 \%, 68.38 \%$ and $49.30 \%$ at 1,3 , and 5 years, respectively (7). In another report from Shiraz Hospitals, the survival accumulation at the end of 1, 3, 5 years were $89 \%$, 71\%, and 57\% that are significantly higher than survival of patients in Yazd city (4). Differences in bladder cancer survival times can be due to differences in socioeconomic conditions, proper screening, and the early diagnosis of the disease using new methods.

In 2012, the number of bladder cancer death in Asia was 524,465 cases. Mortality in men (67.1\%) was more than women (32.9\%) (1). In the present study, the five-year survival rate in Yazdi male and female patients suffering from bladder cancer was 51.5\% and 50\%, respectively, and no significant difference was obtained in survival time between male and female patients $(\mathrm{P}=0.33)$. Compatible with our data, Shamsnia and colleagues did not found a significant difference between sexes of patients and bladder cancer in Shiraz city (7). Also, Rezaianzadeh et al. did not observe any significant difference in the survival rate of Iranian bladder cancer patients based on sex (4). In our study, men and women had the mean survival time of 77.35 and 44.20 months, respectively, but this difference was not significant. In contrast to our results, in some studies women had significantly poorer bladder cancer survival than men (11, 12). A reason for such difference may be the small number of female patients in our study.
The 5-year survival rate in Yazdi patients with bladder cancer showed a significant difference regarding aged groups. The older patients (70 - 89 years vs. 30 - 69 years) had lower survival. In another study reported that the survival rates of the patients in the age group of $60-70$ and 70 - 80 years reduced respectively 1.3 and 1.2 times in comparison to those under 60 years old (13). Results of the present study reveal that the age plays an important role in survival of patients (Cox regression method). Patients in age range of 30-69 year had the risk of bladder cancer 29.3 time more compared to those in the age group 70 - 89 years. In a study conducted by Shamsnia, patients in the age group of 50 - 60 and 60 - 70 years old had the risk of bladder cancer 2.7 and 4.7 times more in comparison to the patients aging under 50 years (7). Consistent with our findings, many reports approved the association of age with survival in patients with bladder cancer $(4,6,7,14)$.

Results of the present study showed that type of cancer treatment is an influential factor in survival of patients. Patients treated by TURBT method had better survival than those who had undergone partial or radical cystectomy. People who received the systemic chemotherapy had significantly a lower survival time comparing to those who were not treated with this method. In a study conducted in Iran (4) and in the study by Gulliford et al. (13), type of cancer therapy was indicated as a significant factor in the survival of patients. Consistent with our findings, in these studies, the chemotherapy was associated with decreased five-year survival and surgery method increased survival time of bladder cancer patients. In the present study, intravesical chemotherapy did not significantly reduced survival time, rather increased it. Several studies have reported that intravesical chemotherapy decreased cancer progression and improved survival (15). Literature data revealed that the 5-year survival of patients suffering from invasive bladder cancer under radiotherapy is $20 \%$ $41 \%(16,17)$. In our study, the 5-year survival of patients treated by radiotherapy is $38 \%$ that is comparable to previous reported data.

In the present study, no significant difference was observed in survival time of bladder cancer patients between urban and rural residents. This showed that the health statuses of urban Yazdi residents was similar to those of rural counterparts. This data is not consistent with other results of cancer research in rural and urban areas worldwide. Pang et al. reported a clear difference in the bladder cancer mortality rates in the urban and rural areas of China (14). In India, cancer survival in rural areas is lower than big cities (15). According to the cancer research findings in Australia, villages had demonstrably the poorer survival when compared to major cities (18). The difference between our findings and above researches may be due to our 


\begin{tabular}{|c|c|c|c|c|c|c|}
\hline \multirow[t]{2}{*}{ Variables } & \multirow[t]{2}{*}{ No. (\%) } & \multicolumn{3}{|c|}{ Survival Rate, \% } & \multirow{2}{*}{ Mean Survival Time \pm SE, Mo } & \multirow[t]{2}{*}{ P Value } \\
\hline & & 1 year & 3 year & 5 year & & \\
\hline Gender & & & & & & 0.33 \\
\hline Male & $300(88.2)$ & 92.5 & 61 & 51.5 & $70.35 \pm 6.42$ & \\
\hline Female & $40(11.8)$ & 91 & 59.5 & 50 & $44.20 \pm 8.75$ & \\
\hline Total & $340(100)$ & 91 & 58 & 51.4 & $68.55 \pm 6.05$ & \\
\hline Age, $y$ & & & & & & 0.005 \\
\hline $30-69$ & $192(53.5)$ & 91.5 & 55 & 42 & $80.78 \pm 7.87$ & \\
\hline $70-89$ & $148(43.5)$ & 91 & 46 & 26 & $50.87 \pm 7.60$ & \\
\hline \multicolumn{7}{|l|}{ place of residence } \\
\hline Urban & $264(77.6)$ & 91.5 & 60 & 36 & $70.73 \pm 6.84$ & 0.55 \\
\hline Rural & $76(22.4)$ & 90 & 55 & 22 & $48.72 \pm 7.06$ & \\
\hline Stage & & & & & & 0.0003 \\
\hline $0+\mathrm{I}$ & $140(41.2)$ & 98 & 86 & 66 & $82.43 \pm 9.19$ & \\
\hline II & $84(24.7)$ & 88 & 42 & 40 & $46.23 \pm 10.08$ & \\
\hline IV & $40(11.8)$ & 100 & 20 & 0 & $26.10 \pm 2.27$ & \\
\hline Unknown & $76(22.4)$ & 97 & 85 & 78 & $85.75 \pm 10.68$ & \\
\hline \multicolumn{7}{|l|}{ Treatment } \\
\hline TURBT & $236(69.4)$ & 98 & 82 & 60 & $82.97 \pm 7.42$ & \\
\hline Partial cystectomy & $28(8.2)$ & 100 & 26 & 0 & $24.14 \pm 4.78$ & 0.0003 \\
\hline Radical cystectomy & $76(22.4)$ & 97 & 41 & 30 & $44.73 \pm 8.79$ & \\
\hline Radiotherapy & $56(16.5)$ & & & & & 0.058 \\
\hline Yes & & 100 & 38 & 38 & $39.25 \pm 7.31$ & \\
\hline No & & 98 & 62 & 52 & $72.53 \pm 6.64$ & \\
\hline Systemic chemotherapy & $116(34.1)$ & & & & & 0.0002 \\
\hline Yes & & 98 & 42 & 30 & $40.84 \pm 6.63$ & \\
\hline No & & 97 & 77 & 59 & $84.56 \pm 7.56$ & \\
\hline Intravesical chemotherapy & $180(54.1)$ & & & & & 0.49 \\
\hline Yes & & 98 & 62 & 54 & $72.04 \pm 8.11$ & \\
\hline No & & 100 & 55 & 42 & $60.89 \pm 7.83$ & \\
\hline
\end{tabular}

\begin{tabular}{|c|c|c|c|}
\hline Variables & Hazard Ratio & Confidence Interval (95\%) & P Value \\
\hline \multicolumn{4}{|l|}{ Age, $y$} \\
\hline $70-89$ & 1 & - & Ref \\
\hline $30-69$ & 29.35 & $7.07-121.72$ & $<0.001$ \\
\hline \multicolumn{4}{|l|}{ Stage } \\
\hline $0+\mathrm{I}$ & 1 & - & Ref \\
\hline II & 1.98 & $0.97-4.04$ & 0.059 \\
\hline IV & 2.93 & $1.28-6.72$ & 0.011 \\
\hline \multicolumn{4}{|l|}{ Treatment } \\
\hline TURBT & 1 & - & Ref \\
\hline Partial cystectomy & 3.59 & $1.53-8.40$ & 0.003 \\
\hline Radical cystectomy & 2.63 & $1.39-4.97$ & 0.003 \\
\hline \multicolumn{4}{|c|}{ Systemic chemotherapy } \\
\hline Yes & 1 & - & Ref \\
\hline No & 0.28 & $0.15-0.51$ & $<0.001$ \\
\hline
\end{tabular}

small sample size or an acceptable health care system in our villages. Cancer survival data from metropolitan and rural areas in Iran are low and further studies are necessary.
In conclusion, the survival rate of patients with bladder cancer in this study was lower than other reports. Since age and stage of bladder cancer are the most important factors in survival time, continuous screening of older peo- 
ple for cancer diagnosis in early stages seemed to improve survival of patients. Intravesical chemotherapy can be the best treatment option for increasing the survival of patients.

\section{Acknowledgments}

None declared.

\section{Footnotes}

Authors' Contribution: None declared.

Conflict of Interest: The authors declare that they have no conflict of interest.

Financial Disclosure: None declared.

\section{References}

1. Pakzad R, Mohammadian-Hafshejani A, Mohammadian M, Pakzad I, Safiri S, Khazaei S, et al. Incidence and Mortality of Bladder Cancer and their Relationship with Development in Asia. Asian Pac J Cancer Prev. 2015;16(16):7365-74. doi: 10.7314/APJCP.2015.16.16.7365. [PubMed: 26514538].

2. Lozano R, Naghavi M, Foreman K, Lim S, Shibuya K, Aboyans V, et al. Global and regional mortality from 235 causes of death for 20 age groups in 1990 and 2010: a systematic analysis for the Global Burden of Disease Study 2010. Lancet. 2012;380(9859):2095-128. doi: 10.1016/S0140-6736(12)61728-0. [PubMed: 23245604].

3. Rudman SM, Crawley D. Pathology and Epidemiology of Cancer. Epidemiology of Bladder Cancer. Springer; 2017. p. 511-22. doi: 10.1007/978-3-319-35153-7_27.

4. Rezaianzadeh A, Mohammadbeigi A, Mobaleghi J, Mohammadsalehi N. Survival analysis of patients with bladder cancer, life table approach. I Midlife Health. 2012;3(2):88-92. doi: 10.4103/09767800.104468. [PubMed: 23372326].

5. Zahedi A, Rafiemanesh H, Enayatrad M, Ghoncheh M, Salehiniya H. Incidence, Trends and Epidemiology of Cancers in North West of Iran. Asian Pac J Cancer Prev. 2015;16(16):7189-93. [PubMed: 26514510]

6. Aliramaji A, Kaseean A, Yousefnia Pasha YR, Shafi H, Kamali S, Safari $\mathrm{M}$, et al. Age distribution types of bladder cancers and their relationship with opium consumption and smoking. Caspian J Intern Med. 2015;6(2):82-6. [PubMed: 26221505].
7. Shamsnia SJ, Khezri AA, Tabbatabaei HR, Mehrabani D. Survival from bladder cancer in Shiraz, Southern Iran: A hospitalbased study. Middle East J Sci Res. 2013;18(4):425-31. doi: 10.5829/idosi.mejsr.2013.18.4.7542.

8. Manterola C, Vial M, Roa JC. Survival of a cohort of patients with intermediate and advanced gall bladder cancer treated with a prospective therapeutic protocol.Acta Cir Bras. 2010;25(3):225-30. doi: 10.1590/S0102-86502010000300003. [PubMed: 20498934].

9. Sankaranarayanan R, Swaminathan R, Jayant K, Brenner H. An overview of cancer survival in Africa, Asia, the Caribbean and Central America: the case for investment in cancer health services. IARC Sci Publ. 2011;(162):257-91. [PubMed: 21675431]

10. Hashibe M, Gao T, Li G, Dalbagni G, Zhang ZF. Comparison of bladder cancer survival among Japanese, Chinese, Filipino, Hawaiian and Caucasian populations in the United States. Asian Pac J Cancer Prev. 2003;4(3):267-73. [PubMed: 14507249].

11. Zaitsu M, Toyokawa S, Tonooka A, Nakamura F, Takeuchi T, Homma Y, et al. Sex differences in bladder cancer pathology and survival: analysis of a population-based cancer registry. Cancer Med. 2015;4(3):36370. doi: 10.1002/cam4.379. [PubMed: 25533611].

12. Horstmann M, Witthuhn R, Falk M, Stenzl A. Gender-specific differences in bladder cancer: a retrospective analysis. Gend Med. 2008;5(4):385-94. doi: 10.1016/j.genm.2008.11.002. [PubMed: 19108811].

13. Gulliford MC, Petruckevitch A, Burney PG. Survival with bladder cancer, evaluation of delay in treatment, type of surgeon, and modality of treatment. BMJ. 1991;303(6800):437-40. doi: 10.1136/bmj.303.6800.437. [PubMed: 1912834].

14. Pang C, Guan Y, Li H, Chen W, Zhu G. Urologic cancer in China. Jpn J Clin Oncol. 2016;46(6):497-501. doi: 10.1093/jjco/hyw034. [PubMed: 27049022].

15. Chung F, Liao P, Sun Y, Amirshahi B, Fazel H, Shapiro CM, et al. Perioperative practical experiences in using a level 2 portable polysomnography. Sleep Breath. 2011;15(3):367-75. doi: 10.1007/s11325-010-0340-9. [PubMed: 20232260].

16. Greven KM, Solin LJ, Hanks GE. Prognostic factors in patients with bladder carcinoma treated with definitive irradiation. Cancer. 1990;65(4):908-12. doi: 10.1002/1097-0142(19900215)65:4lt;908::AIDCNCR2820650415gt;3.0.CO;2-Z. [PubMed: 2404564].

17. McBain CA, Logue JP. Radiation therapy for muscle-invasive bladder cancer: treatment planning and delivery in the 21st century. Semin Radiat Oncol. 2005;15(1):42-8. doi: 10.1016/j.semradonc.2004.07.015. [PubMed: 15662606].

18. Baharlou R, Ahmadi Vasmehjani A, Dehghani A, Ghobadifar MA Khoubyari M. Reduced interleukin-17 and transforming growth factor Beta levels in peripheral blood as indicators for following the course of bladder cancer. Immune Netw. 2014;14(3):156-63. doi: 10.4110/in.2014.14.3.156. [PubMed: 24999312]. 